01

\title{
Влияние фторзаместителей на параметры внутри- и межмолекулярного взаимодействия молекул 1,4-дистирилбензола
}

\author{
(C) В.В. Компанеец ${ }^{1}$, К.Р. Каримуллин ${ }^{1,2}$, И.А. Васильева ${ }^{1}$ \\ ${ }^{1}$ Московский педагогический государственный университет, \\ 119435 Москва, Россия \\ ${ }^{2}$ Институт спектроскопии РАН, \\ 108840 Москва, Троицк, Россия \\ e-mail: kompaneez@gmail.com
}

Поступила в редакцию 11.12.2018 г.

В окончательной редакции 25.06.2019 г.

Принята к публикации 08.07.2019 г.

Проведен сравнительный анализ параметров франк-кондоновского (FC) и герцберг-теллеровского (НT) взаимодействий, формирующих тонкоструктурные спектры стильбена, 1,4-дистирилбензола и тетрафтордистерилбензола, близких по химической структуре, но отличающихся длиной $\pi$-сопряжения и наличием заместителей в бензольных кольцах. Получены численные значения константы не только внутримолекулярного взаимодействия FC, но одновременно определено значение НТ-параметра как количественное значение проекции вектора дипольного момента электронного перехода на нормальные колебательные координаты. Решен вопрос о переносимости этих параметров в гомологическом ряду молекул стильбена, содержащих одинаковые наборы структурных элементов, что позволяет расширить применение фрагментарного подхода для описания фундаментальных полос органических молекул разных гомологических рядов и решает проблему изучения структуры спектров больших молекул.

Ключевые слова: спектры флуоресценции, вибронная полоса, бесфононная линия, фононное крыло, внутри- и межмолекулярное взаимодействия.

DOI: $10.21883 /$ OS.2019.11.48503.361-18

\section{Введение}

Методы селективной оптической спектроскопии, такие как исследование сопряженных спектров люминесценции $[1,2]$, фотонное эхо [3,4], спектроскопия одиночных молекул $[5,6]$ и другие, широко используются в последнее время для исследования спектральных и фотофизических свойств различных люминесцентных материалов [7]. Актуальной задачей в данной области является поиск и характеризация новых люминофоров и маркеров [8]. Исследования оптических и фотофизических свойств олигомерных и полимерных $\pi$-конъюгированных органических соединений особенно интенсивно ведутся последние несколько лет. В качестве примера можно отметить исследования полифениленвинилена (ППВ), обладающего уникальными электролюминесцентными свойствами, область практического применения которого постоянно растет. Структурной частью подобных соединений часто являются стильбены и дистирилбензолы. В связи с этим появляется много теоретических и экспериментальных работ, посвященных исследованию спектрально-люминесцентных свойств транс-стильбена (СБ) [9-12], 1,4-дистирилбензола (ДСБ) $[13,14]$ и $\alpha, \omega$-тетрафтордистирилбензола (ФДСБ) [15-17].

В большинстве работ, посвященных моделированию сложных молекул, авторы опираются на теоретические методы, исключающие экспериментальные исследова- ния молекулярных тонкоструктурных электронно-колебательных (вибронных) спектров [18-22]. Такие методы позволяют рассчитывать колебательную структуру спектров молекул, но очень трудоемки и вследствие большой погрешности вычислений неприменимы для больших молекул в возбужденных состояниях, что делает невозможным получение сведений о тонких эффектах электрон-электронных и электрон-ядерных взаимодействий.

Наиболее точно энергию основного и возбужденного состояний молекул можно определить, используя теорию возмущения Меллера-Плессета второго порядка MP2 или теорию функционала плотности DFT с базисом B3LYP [23-26]. Однако эти методы наиболее затратны как по ресурсам компьютера, так и по времени. Из всех остальных теоретических методов компромиссными между временем счета и точностью являются параметрические методы.

В существующих на сегодняшний день методах расчета вибронных параметров колебательной структуры спектров многоатомных молекул используется либо франк-кондоновское (FC), либо герцберг-теллеровское (НТ) приближения. Поэтому актуальной задачей становится возможность провести вибрационный анализ сопряженных спектров флуоресценции и поглощения замещенных дифенилполиенов и получить численное значение константы не только внутримолекулярного взаимодействия $\mathrm{FC}$, но одновременно определить значение НТ-параметра как значения проекции 
вектора дипольного момента электронного перехода на нормальные колебательные координаты. Также представляет интерес вопрос о динамике изменений значений этих параметров в гомологическом ряду молекул, содержащих одинаковые наборы структурных элементов. Это позволило бы расширить применение фрагментарного подхода для описания фундаментальных полос органических молекул разных гомологических рядов и решить проблему изучения структуры спектров больших молекул.

В динамической теории электрон-фононных оптических полос, разработанной Осадько [27], учитывается влияние на форму оптических полос одновременно FCи НТ-взаимодействий. При учете обоих взаимодействий сложной задачей становится даже расчет одноквантовых амплитуд. Интегральная интенсивность любой вибронной линии сопряженного спектра с учетом проявления в нем обоих взаимодействий определяется как сумма двух слагаемых:

$$
I(\omega)=I_{\text {БФЛ }}(\omega)+I_{\text {ФК }}(\omega) .
$$

Первое слагаемое в данной формуле описывает фотопереход без рождения фононов - бесфононную линию (БФЛ). Форма БФЛ при этом определяется функцией Лоренца. Второе слагаемое описывает фотопереходы с рождением одного, двух и более фононов. Совокупность всех электрон-фононных переходов, описываемых этим слагаемым, называется фононным крылом (ФК).

Известно, что структура поглощения и флуоресценции ряда молекул (например, полиенов и дифенилполиенов), их стереоизомеров, а также замещенных форм часто имеют очень похожие формы [28]. Различия в интенсивности основных вибронных линий (10\%) сопоставимы с погрешностью их расчета. Таким образом, интерпретация и анализ таких спектров и, что более важно, идентификация соединений (в случае смесей) на основе спектральных данных является чрезвычайно важной задачей, поскольку дает возможность изучать спектры одних соединений (более простых или доступных) и прогнозировать спектры других (сложных).

Представляет особый интерес переносимость параметров в гомологическом ряду молекул, содержащих фрагменты разного типа. В связи с этим появляется много теоретических и экспериментальных работ, посвященных спектрально-люминесцентным свойствам транс-стильбена (СБ) [29-32], 1,4-дистирилбензола (ДСБ) $[33,34]$ и $\alpha, \omega$-тетрафтордистирилбензола (ФДСБ) [15,35,36]. Молекулу ДСБ формально можно получить присоединением в пара-положении транс-СБ фенильного кольца с еще одной этиленовой связью $(-\mathrm{CH}=\mathrm{CH}-\mathrm{Ph})$, т.е. она представляет собой как бы сдвоенную молекулу стильбена. Молекула ФДСБ отличается от молекулы дистирилбензола тем, что атомы Н в бензольных кольцах ДСБ заменяют атомы F. Известно, что заместители в ароматических кольцах молекул дифенилполиенов влияют на перераспределение электронной плотности в молекуле [37-39]. Поэтому возможно провести сравнительный анализ спектральных свойств и оценки вклада внутримолекулярных взаимодействий в формирование тонкоструктурных спектров стильбена (СБ) и 1,4-дистирилбензола (ДСБ), и тетрафтордистерилбензола, близких по химической структуре, но отличающихся длиной $\pi$-сопряжения и наличием заместителей в бензольных кольцах.

\section{Экспериментальная часть}

Объектами исследования в настоящей работе являются параметры внутри- и межмолекулярного взаимодействий молекул, структурные формулы которых показаны на рис. 1.

Тонкоструктурные сопряженные спектры флуоресценции и возбуждения флуоресценции СБ, ДСБ и ФДСБ приведены на рис. 2-4.

Спектры ДСБ сдвинуты относительно спектров СБ на $\sim 60 \mathrm{~cm}^{-1}$ в длинноволновую область. Между сопряженными спектрами обоих соединений наблюдается относительно небольшое нарушение зеркальной симметрии как по распределению интенсивности, так и по частотам.

Аналогичный спектральный сдвиг существует и между спектрами ДСБ и ФДСБ. Тонкая структура спектров всех трех соединений проявляется на сплошном, интенсивном фоне, самый большой фон наблюдается в спектрах ФДСБ. Тем не менее частоты, проявляющиеся в сопряженных спектрах флуоресценции и возбуждения флуоресценции всех трех веществ, близки, а значит, сопоставимы.

Процесс обработки экспериментальных спектров можно описать в несколько этапов. На первом этапе из полученных экспериментально спектров находятся значения частот и интегральных интенсивностей локальных максимумов на сопряженных спектрах. Впоследствии полученные частоты определяют положение БФЛ на моделированных спектрах. На втором этапе происходит модельное воссоздание вибронных полос на найденных в первом этапе положениях частот. На этом этапе учитывается, что вибронная полоса состоит из БФЛ, моделируемой функцией Лоренца, и ФК, моделируемого функцией Гаусса. Учитывается также, что ширина БФЛ растет с увеличением номера вибронного уровня. В процессе моделирования оптимизируются ширины БФЛ и ФК, расстояние между максимумами БФЛ и ФК в шкале частот, а также значения фактора Дебая-Валера до тех пор, пока моделируемый спектр не совпадет с экспериментальным. Результатом второго этапа является получение истинных значений относительных интегральных интенсивностей вибронных полос. Полученные в результате моделирования относительные интегральные интенсивности вибронных полос, а также уточненные колебательные частоты позволяют с большой достоверностью определять нормальные колебания и проводить вибрационный анализ [40-42]. 
Таблица 1. Интерпретация колебаний молекул СБ, ДСБ и ФДСБ по основным частотам $\left(\mathrm{cm}^{-1}\right)$, в скобках приведены частоты, проявляющиеся в спектрах возбуждения флуоресценции

\begin{tabular}{|c|c|c|c|c|}
\hline Соединение & СБ & ДСБ & ФДСБ & Интерпретация колебаний \\
\hline $\begin{array}{l}\text { Положение полосы } \\
v_{0-0}-v_{i}\left(v_{i}-v_{0-0}\right)\end{array}$ & $\begin{array}{c}321 \\
(320)\end{array}$ & $\begin{array}{c}304 \\
(304)\end{array}$ & 308 & \multirow{3}{*}{ Изгиб по $\mathrm{Ce}=\mathrm{Ce}-\mathrm{Cph}-$ связи } \\
\hline Параметр FC $a / 2$ & 0.38 & 0.39 & 0.23 & \\
\hline Параметр НТ $\alpha$ & 0.1 & 0.07 & 0.13 & \\
\hline $\begin{array}{l}\text { Положение полосы } \\
v_{0-0}-v_{i}\left(v_{i}-v_{0-0}\right)\end{array}$ & $\begin{array}{c}422 \\
(401)\end{array}$ & $(410)$ & $\begin{array}{c}422 \\
(422)\end{array}$ & \multirow{3}{*}{ Деформация колец } \\
\hline Параметр FC $a / 2$ & 0.59 & 0.16 & 0.55 & \\
\hline Параметр НТ $\alpha$ & 0.06 & 0.06 & 0.04 & \\
\hline $\begin{array}{l}\text { Положение полосы } \\
v_{0-0}-v_{i}\left(v_{i}-v_{0-0}\right)\end{array}$ & $(682)$ & $\begin{array}{c}674 \\
(681) \\
\end{array}$ & $\begin{array}{c}696 \\
(696) \\
\end{array}$ & \multirow[t]{3}{*}{ Изгиб C-Н-связей колец } \\
\hline Параметр FC $a / 2$ & 0.23 & 0.32 & 0.38 & \\
\hline Параметр НТ $\alpha$ & -0.13 & -0.22 & -0.1 & \\
\hline $\begin{array}{l}\text { Положение полосы } \\
v_{0-0}-v_{i}\left(v_{i}-v_{0-0}\right)\end{array}$ & $\begin{array}{c}748 \\
(742)\end{array}$ & $(732)$ & $\begin{array}{c}750 \\
(750) \\
\end{array}$ & \multirow[t]{3}{*}{ Изгиб C-Н-связей колец } \\
\hline Параметр FC $a / 2$ & 0.30 & 0.16 & 0.18 & \\
\hline Параметр НТ $\alpha$ & 0.05 & 0.06 & 0.04 & \\
\hline $\begin{array}{l}\text { Положение полосы } \\
v_{0-0}-v_{i}\left(v_{i}-v_{0-0}\right)\end{array}$ & $\begin{array}{l}1014 \\
(993)\end{array}$ & $\begin{array}{l}1008 \\
(996)\end{array}$ & $\begin{array}{c}1021 \\
(1011)\end{array}$ & \multirow[t]{3}{*}{ Изгиб C-Н-связей колец и C-C-С-связей } \\
\hline Параметр FC $a / 2$ & 0.48 & 0.36 & 0.31 & \\
\hline Параметр НТ $\alpha$ & -0.02 & -0.03 & -0.14 & \\
\hline $\begin{array}{l}\text { Положение полосы } \\
v_{0-0}-v_{i}\left(v_{i}-v_{0-0}\right)\end{array}$ & $\begin{array}{c}1088 \\
(1066) \\
\end{array}$ & 1088 & $\begin{array}{c}1060 \\
(1057)\end{array}$ & \multirow[t]{3}{*}{ Изгиб С-H- и Се-Cph-связей } \\
\hline Параметр FC $a / 2$ & 0.38 & 0.15 & 0.41 & \\
\hline Параметр НТ $\alpha$ & 0.05 & 0.05 & 009 & \\
\hline $\begin{array}{l}\text { Положение полосы } \\
v_{0-0}-v_{i}\left(v_{i}-v_{0-0}\right)\end{array}$ & $\begin{array}{c}1187 \\
(1187)\end{array}$ & $\begin{array}{c}1187 \\
(1181)\end{array}$ & 1182 & \multirow[t]{3}{*}{ Изгиб C-H и растяжение $\mathrm{Ce}-\mathrm{Cph-связей}$} \\
\hline Параметр FC $a / 2$ & 0.51 & 0.49 & 0.30 & \\
\hline Параметр НТ $\alpha$ & -0.09 & -0.04 & -0.20 & \\
\hline $\begin{array}{l}\text { Положение полосы } \\
v_{0-0}-v_{i}\left(v_{i}-v_{0-0}\right)\end{array}$ & 1213 & 1209 & $\begin{array}{c}1201 \\
(1201)\end{array}$ & \multirow[t]{3}{*}{ Изгиб С.Н и растяжение Се.Сph- связей } \\
\hline Параметр FC a/2 & 0.21 & 0.25 & 0.37 & \\
\hline Параметр НТ $\alpha$ & -0.11 & -0.15 & -0.13 & \\
\hline $\begin{array}{l}\text { Положение полосы } \\
v_{0-0}-v_{i}\left(v_{i}-v_{0-0}\right)\end{array}$ & $(1261)$ & $\begin{array}{c}1250 \\
(1265) \\
\end{array}$ & $\begin{array}{c}1250 \\
(1249) \\
\end{array}$ & \multirow[t]{3}{*}{ Изгиб C-H и растяжение $\mathrm{Ce}-\mathrm{Cph}-$ связей } \\
\hline Параметр FC $a / 2$ & 0.34 & 0.41 & 0.53 & \\
\hline Параметр НТ $\alpha$ & 0.20 & -0.04 & -0.02 & \\
\hline $\begin{array}{l}\text { Положение полосы } \\
v_{0-0}-v_{i}\left(v_{i}-v_{0-0}\right) \\
\end{array}$ & 1337 (1335) & $(1330)$ & $1333(1323)$ & \multirow[t]{3}{*}{ Изгиб $\mathrm{C}-\mathrm{H}$ и $\mathrm{Ce}-\mathrm{Cph}-$ связей } \\
\hline Параметр FC $a / 2$ & 0.48 & 0.29 & 0.39 & \\
\hline Параметр НТ $\alpha$ & -0.05 & 0.19 & -0.22 & \\
\hline
\end{tabular}


Таблица 1 (продолжение).

\begin{tabular}{|c|c|c|c|c|}
\hline Соединение & СБ & ДСБ & ФДСБ & Интерпретация колебаний \\
\hline $\begin{array}{l}\text { Положение полосы } \\
v_{0-0}-v_{i}\left(v_{i}-v_{0-0}\right)\end{array}$ & 15321553 & (1525) 1540 & $(1540)$ & \multirow[t]{3}{*}{ Растяжение $\mathrm{Cph}-\mathrm{Cph-связей}$} \\
\hline Параметр FC a/2 & 0.26 & 0.38 & 0.56 & \\
\hline Параметр НТ $\alpha$ & -0.16 & 0.04 & -0.32 & \\
\hline $\begin{array}{l}\text { Положение полосы } \\
v_{0-0}-v_{i}\left(v_{i}-v_{0-0}\right)\end{array}$ & 1603 & $(1608) 1600(1610)$ & $1600(1600)$ & \multirow[t]{3}{*}{ Растяжение $\mathrm{Ce}-\mathrm{Cph}$-связей колец } \\
\hline Параметр FC $a / 2$ & 0.70 & 0.62 & 0.58 & \\
\hline Параметр НТ $\alpha$ & 0.02 & -0.11 & -0.13 & \\
\hline $\begin{array}{l}\text { Положение полосы } \\
v_{0-0}-v_{i}\left(v_{i}-v_{0-0}\right)\end{array}$ & 1653 & $1657(1668)$ & $1638(1642)$ & \multirow[t]{3}{*}{ Растяжение олефиновых связей } \\
\hline Параметр FC $a / 2$ & 0.43 & 0.39 & 0.72 & \\
\hline Параметр НТ $\alpha$ & -0.33 & 0.09 & -0.13 & \\
\hline
\end{tabular}
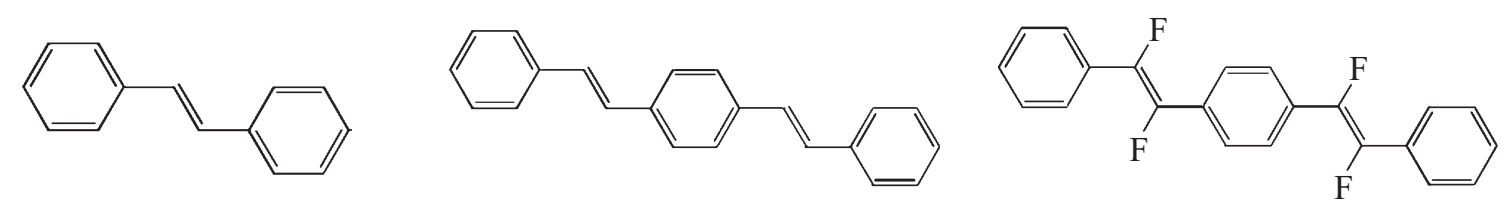

Рис. 1. Слева направо: молекулы транс-стильбена, 1,4-дистирилбензола и $\alpha, \omega$-тетрафтордистирилбензола.

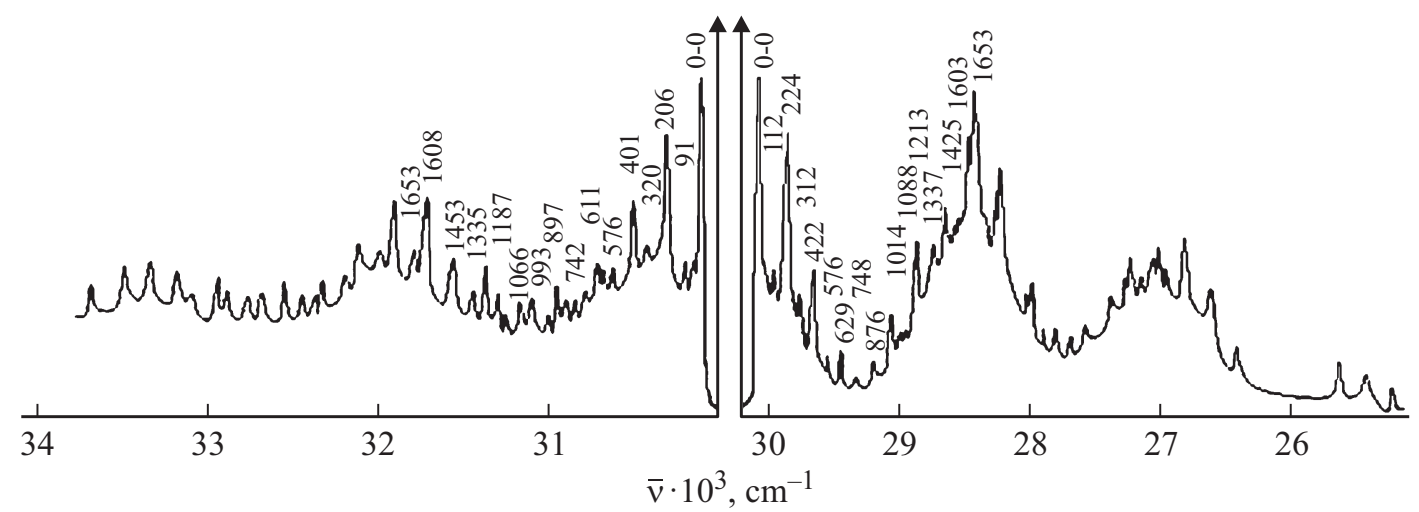

Рис. 2. Спектры флуоресценции (слева) и возбуждения флуоресценции (справа) транс-стильбена в октане, концентрация $3 \cdot 10^{-8}$ моль/л при $4.2 \mathrm{~K}$.

Относительные интенсивности полос в сопряженных спектрах, а также параметры $a$ и $\alpha$ для молекулы СБ были рассчитаны нами по вышеописанной методике. Совпадение экспериментального и моделируемого спектров СБ удалось получить при ширинах БФЛ $12 \mathrm{~cm}^{-1}$, ФК $144 \mathrm{~cm}^{-1}$ и факторе Дебая-Валера, равном 0.2. Малая величина фактора Дебая-Валера позволяет говорить о сильном электрон-фононном взаимодействии молекул стильбена с матрицей растворителя. Параметры были рассчитаны для полос, соответствующих колебаниям в интервале частот от 0 до $1653 \mathrm{~cm}^{-1}$. Достоверность полученных параметров проверялась расчетом относительной интенсивности полос, соответствующих обертонам и комбинациям частот проявляющихся нормальных колебаний. Нами были получены сводные таблицы частот и интенсивностей вибронных полос для СБ, ДСБ и ФДСБ [37-40]. Полученные результаты качественно согласуются с результатами расчетов, выполненных чисто теоретическими и полуэмпирическими методами в работах [43-46]. На основании этих данных в настоящей работе исследована возможность применения фрагментарного подхода к этим молекулам. 


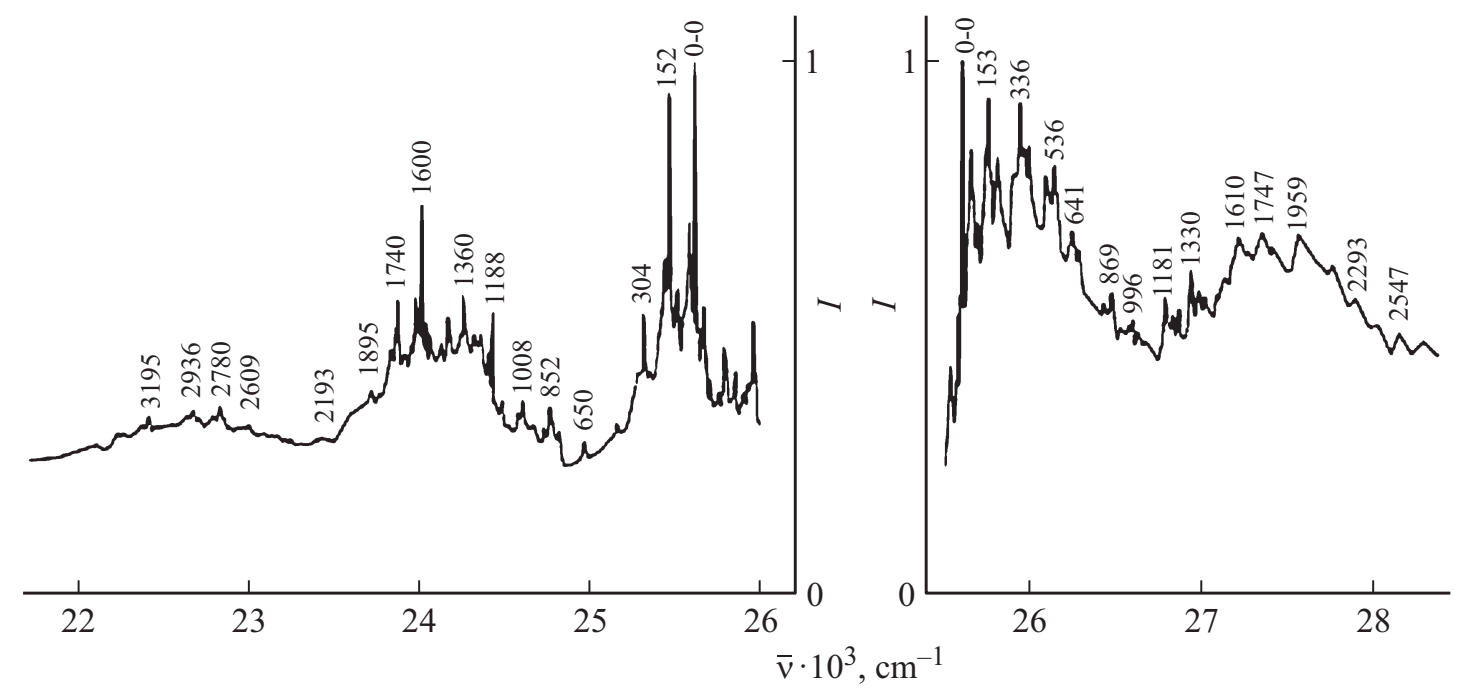

Рис. 3. Спектры флуоресценции (слева) и возбуждения флуоресценции (справа) 1,4-дистирилбензола в $н$-гексане концентрации $C=10^{-5} \mathrm{M} /$ л при $4.2 \mathrm{~K}[42]$.

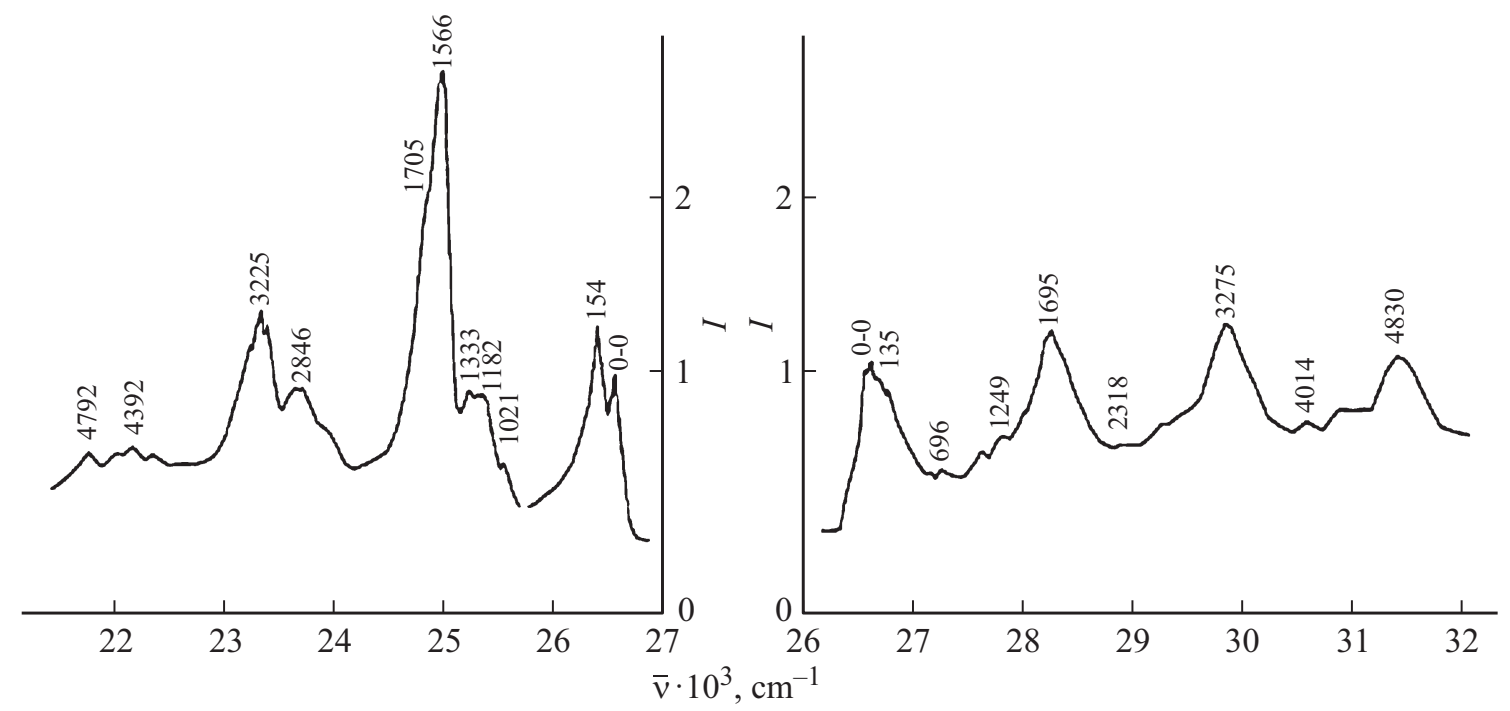

Рис. 4. Спектры флуоресценции (слева) и возбуждения флуоресценции (справа) $\alpha$, $\omega$-тетрафтордистирилбензола в $H$-гексане концентрации $C=10^{-5} \mathrm{M} /$ л при $4.2 \mathrm{~K}[42]$.

\section{Обсуждение результатов}

Как и в случае других исследуемых нами соединений, незеркальность, наблюдаемая между сопряженными спектрами ДСБ как по частотам, так и по распределению интенсивностей по спектрам, может быть объяснена интерференцией FC- и НТ-взаимодействий. Так, некоторые характеристические полосы СБ, такие как, например, 849 и $1684 \mathrm{~cm}^{-1}$, проявляются только в спектре возбуждения флуоресценции, а другие полосы, например $1653 \mathrm{~cm}^{-1}$, только в спектре флуоресценции. Для ДСБ таких полос становится больше. Для ФДСБ количество незеркальных полос также велико (табл. 1).

В сложных органических молекулах, включающих в себя несколько циклических фрагментов, значительная часть колебаний характеризуется одновременным изменением всех $\mathrm{C}-\mathrm{C}$-связей или углов, при этом частоты колебаний различаются мало. В то же время формы колебаний на отдельных циклических фрагментах обладают определенной устойчивостью, т.е. в большинстве случаев меняются при введении заместителей или включении фрагмента в состав большой молекулы. Такая устойчивость позволяет говорить о линиях СБ, подразумевая, что равные им по частоте линии ДСБ описывают аналогичное состояние подобной СБ молекулы (табл. 2).

Из 24 нормальных колебаний, проявляющихся в спектрах СБ в спектрах ДСБ, можно наблюдать полосы, соответствующие 33 колебаниям, а в спектрах ФДСБ только 25 колебаниям. Практически у всех пар виброн- 
Таблица 2. Диаграмма колебаний параметров Франка-Кондона и Герцберг-Теллера для СБ, ДСБ и ФДСБ

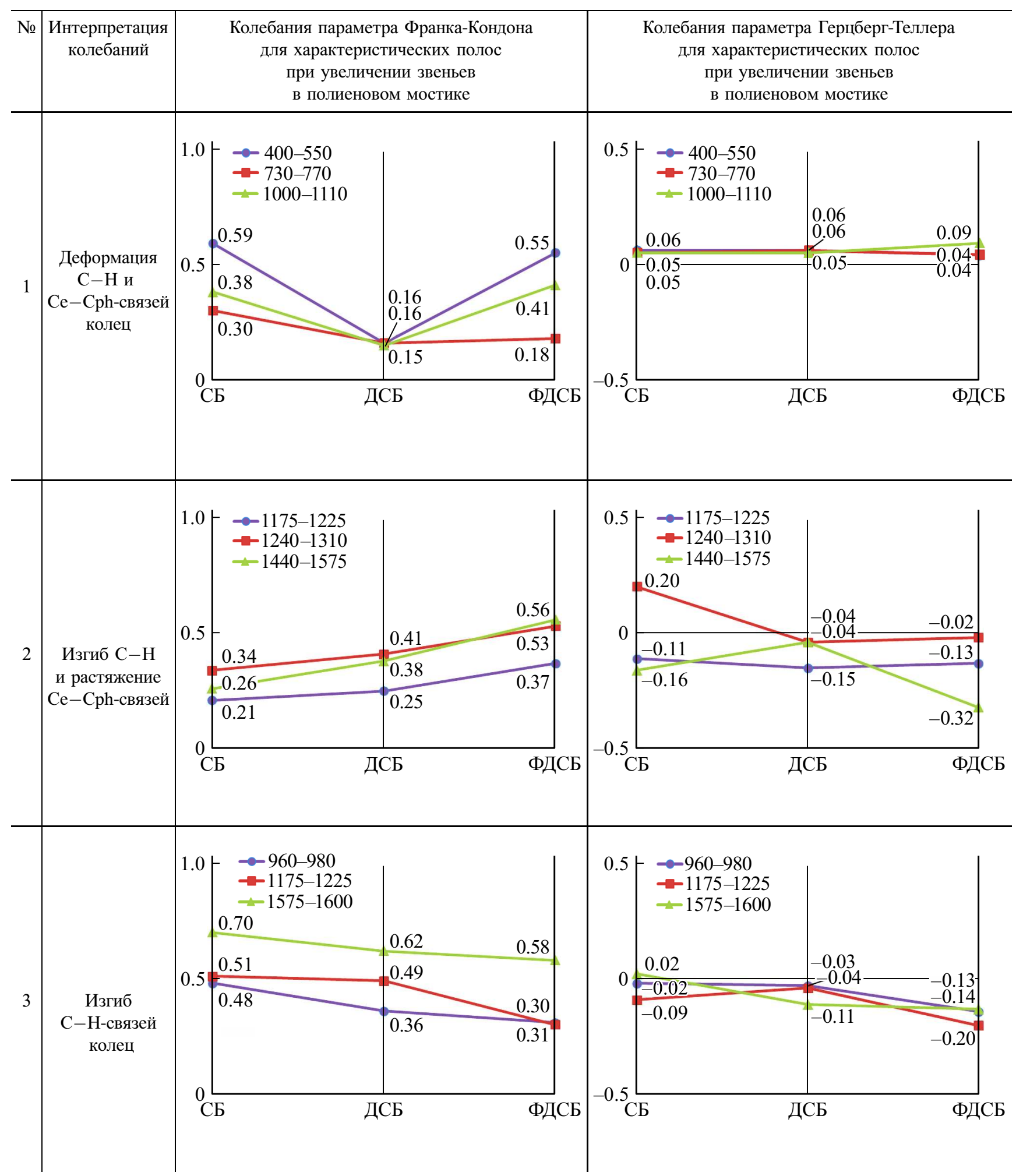

ных полос в сопряженных спектрах, обусловленных одинаковыми для обеих молекул колебаниями, параметры внутримолекулярных взаимодействий отличаются, что приводит к различию в распределении интенсивности по сопряженным спектрам СБ, ДСБ и ФДСБ.
На группах полос (табл. 2, п. 1) наблюдается деформация С-Н-связей и олефиновых связей колец. При этом в ряду СБ-ДСБ-ФДСБ мы наблюдаем, что энергия межмолекулярного взаимодействия (параметр FC) претерпевает скачок у ДСБ, но в то же время практически 
Таблица 2 (продолжение).

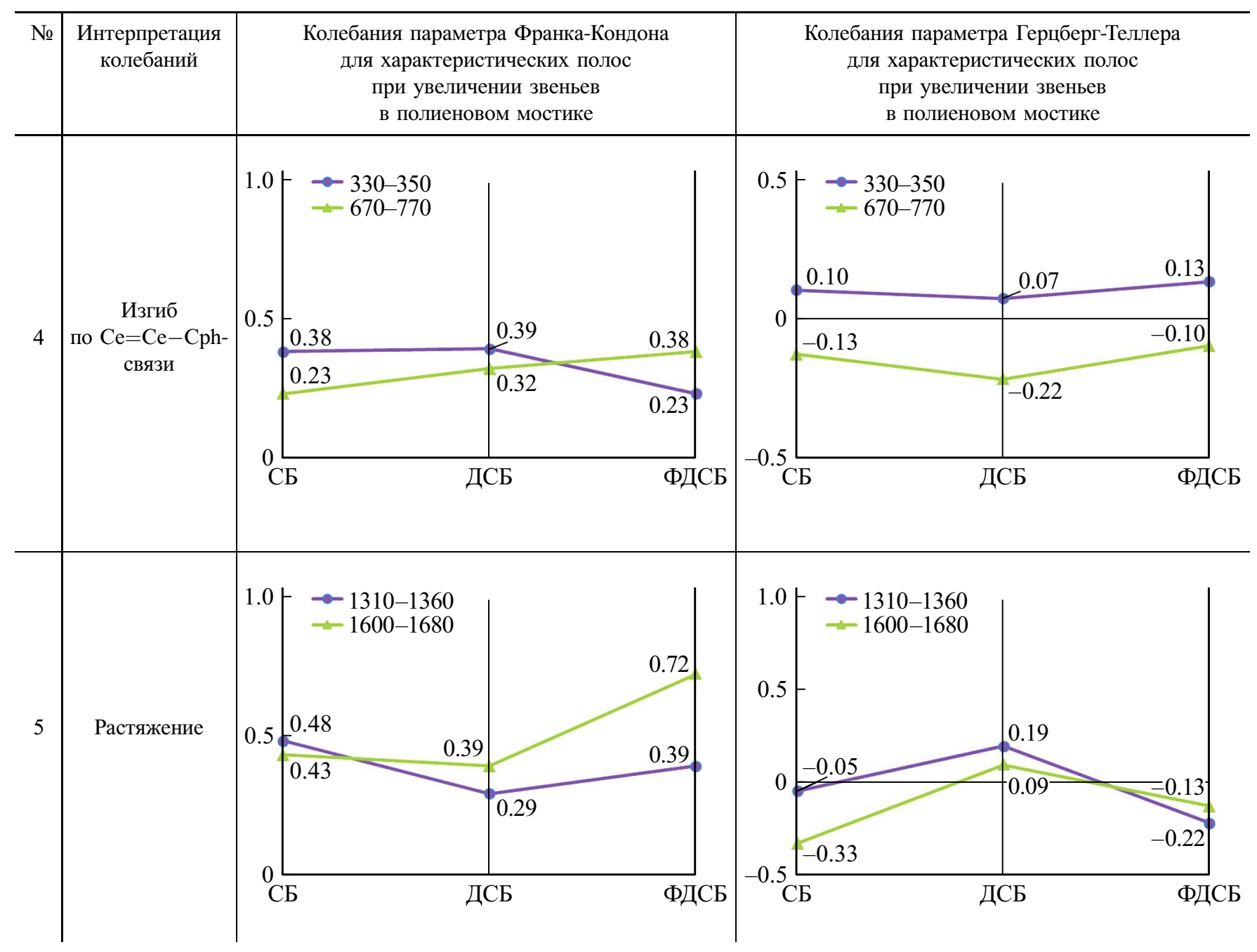

одинакова у СБ и ФДСБ, а энергия внутримолекулярного взаимодействия (параметр НТ) остается неизменной для всех трех соединений. Это можно объяснить уменьшением числа степеней свободы при присоединении третьего кольца и улучшением энергетической стабильности молекулы при фторировании колец.

Похожую реакцию FC- и НТ-параметров можно наблюдать также на другой группе полос (табл. 2, п. 3), когда происходит только изгиб С-Н-связей колец. Наблюдается незначительное уменьшение обоих параметров, причем параметр НТ падает быстрее параметра FC. При этом на характеристической частоте колец $1600 \mathrm{~cm}^{-1}$ наблюдется значительное падение при присоединении третьего кольца, а фторирование практически не оказывает влияния на параметры. В то же самое время на полосах 980 и $1175 \mathrm{~cm}^{-1}$ больший эффект производит присоединение фтора.

Введение фтора в свободные фенильные кольца молекулы ДСБ приводит к перераспределению электронной плотности равномерно по молекуле, поскольку акцептор, фтор, стягивает на себя электронную плотность, отданную кольцом. Таким образом, стянутые фтором электроны, с одной стороны, приводят к повышению энергии молекулы, а с другой - уменьшают дипольный момент молекулы и стабилизируют ее.

С другой стороны, рост молекулярной массы и длины молекулы, а также увеличение числа ее конформаций вызывает незначительный прирост параметров, отвечающих за межмолекулярное взаимодействие (табл. 2, пп. 2,5) на полосах, например 1240-1310 и $1600-1680 \mathrm{~cm}^{-1}$

Пока деформация или растяжение присутствуют только в полиеновой цепочке у двойных связей (область частот ниже $1000 \mathrm{~cm}^{-1}$ ), это не сильно отражается на характеристиках молекулы. Этим можно объяснить практически равные параметры при некоторых частотах (табл. 2, п. 4).

В области частот выше $1000 \mathrm{~cm}^{-1}$ у молекул наблюдается изгиб $\mathrm{C}-\mathrm{H}$-связей. $\mathrm{B}$ интервале частот $1310-1680 \mathrm{~cm}^{-1}$ изгиб передается на С-С-связи полиеновой цепи, переходя в растяжение валентных связей. У молекул это выражается ростом параметра внутри- 
молекулярного взаимодействия (FC) и ростом по модулю параметра межмолекулярного взаимодействия (НT). Молекула ФДСБ, отягощенная с одной стороны тремя ароматическими кольцами, а с другой стороны фтором, дает больший скачок в энергии внутримолекулярного взаимодействия при приближении к характеристической частоте $1650 \mathrm{~cm}^{-1}$.

\section{Заключение}

Для изучения влияния на форму оптических полос FC- и НТ-взаимодействий в настоящее время широко используются полуэмпирические и теоретические методы (например, MP2, DFT с базисом B3LYP). Однако не теряют актуальности поиски частных решений. Используемый в работе метод позволяет проводить вибронный анализ сопряженных спектров флуоресценции поглощения органических примесных молекул в парафиновых матрицах в рамках теории электронно-колебательного взаимодействия. Проведенное исследование показывает, что полученные данным методом параметры внутри- и межмолекулярного взаимодействий, формирующие тонкоструктурные спектры выбранных молекул, демонстрируют высокую степень переносимости в гомологическом ряду молекул и возможность применения фрагментарного подхода при описании фундаментальных полос в сложных органических молекулах, содержащих полиеновые цепочки и фенильные кольца.

Теоретическое моделирование подтверждает, что введение в дифенилполиеновые кольца электронно-акцепторного заместителя приводит к уширению линий и повышению фона спектров, что хорошо коррелирует с ранее опубликованными работами, выполненными другими методами.

\section{Финансирование работы}

Данное исследование выполнено при поддержке Российского фонда фундаментальных исследований и Правительства Москвы (проект № 19-32-70005-мол_а_мос)

\section{Конфликт интересов}

Авторы заявляют, что у них нет конфликта интересов.

\section{Список литературы}

[1] Магарян К.А., Михайлов М.А., Каримуллин К.Р., Васильева И.А., Климушева Г.В. // Изв. РАН. Сер. физ. 2014. T. 78. № 12. С. 1629. doi 10.7868/S0367676514120199; Magaryan K.A., Mikhailov M.A., Vasilieva I.A., Karimullin K.R., Klimusheva G.V. // Bull. RAS: Physics. 2014. V. 78. N 12. P. 1336. doi $10.3103 / \mathrm{S} 1062873814120193$

[2] Magarian K.A., Fedyanin V.V., Karimullin K.R., Vasilieva I.A., Klimusheva G.V. // J. Phys. Conf. Ser. 2013. V. 478. Art. N 012007. doi 10.1088/1742-6596/478/1/012007
[3] Knyazev M.V., Karimullin K.R., Naumov A.V. // Phys. Stat. Sol. (RRL). 2017. V. 11. N 3. Art. N 1600414. doi 10.1002/pssr.201600414

[4] Karimullin K., Knyazev M., Eremchev I., Vainer Y., Naumov A. // Measurement Science and Technology. 2013. V. 24. N 2. Art. N 027002. doi 10.1088/09570233/24/2/027002

[5] Савостьянов А.О., Еремчев И.Ю, Горшелев А.А., Наумов А.В., Старухин А.С. // Письма в ЖЭТФ. 2018. T. 107. № 7. C. 426. doi 10.7868/S0370274X18070044; Savostianov A.O., Eremchev I.Yu, Gorshelev A.A., Naumov A.V., Starukhin A.S. // JETP Lett. 2018. V. 107. N 7. P. 406. doi 10.1134/S002136401807007X

[6] Naumov A.V., Gorshelev A.A., Gladush M.G., Anikushina T.A., Golovanova A.V., Köhler J., Kador L. // Nano Lett. 2018. V. 18. N 10. P. 6129. doi 10.1021/acs.nanolett.8b01753

[7] Наумов А.В. // УФН. 2013. Т. 183. № 6. С. 633. doi 10.3367/UFNr.0179.200903n.0322; Naumov A.V. // Physics - Uspekhi. 2013. V. 56. N 6. P. 605. doi 10.3367/UFNe.0183.201306f.0633

[8] Karimullin K.R., Naumov A.V. // J. Luminesc. 2014. V. 152. P. 15. doi 10.1016/j.jlumin.2014.01.069

[9] Zhang A., Xiao Ch., Wu Ya., Li Ch., Ji Yu., Li L., Hu W., Wang Zh., Ma W., Li W. // Macromolecules. 2016. V. 49. N 17. P. 6431. doi 10.1021/acs.macromol.6b01446

[10] Katoh R., Hashimoto M., Takahashi A., Sonoda Y., Yago T., Wakasa M. // J. Phys. Chem. C. 2017. V. 121. N 46. P. 25666. doi 10.1021/acs.jpcc.7b06905

[11] Sonoda Y., Goto M., Tsuzuki S., Tamaoki N. // J. Phys. Chem. A. 2007. V. 111. N 51. P. 13441. doi 10.1021/jp0766104

[12] Weigel A., Ernsting N.P. // J. Phys. Chem. B. 2010. V. 114. N 23. P. 7879. doi 10.1021/jp100181z

[13] Marri E., Pannacci D., Galiazzo G., Mazzucato U., Spalletti A. // J. Phys. Chem. A. 2003. V. 107. N 50. P. 11231. doi 10.1021/jp035401q

[14] Gierschner J., Mack H.-G., Lüer L., Oelkrug D. // J. Chem. Phys. 2002. V. 116. N 19. P. 8596. doi 10.1063/1.1469612

[15] Voytova N.A.. Vasil'eva I.A., Nurmuhametov R.N. // Abstracts of Reports XV-th Symposium on High Resolution Molecular Spectroscopy (HighRus-2006), July 18-21, 2006. P. 128.

[16] Buch T.E., Scott G.W. // J. Chem. Phys. 1981. V. 85. N 2. P. 144. doi $10.1021 / \mathrm{j} 150602 \mathrm{a} 008$

[17] Erckel R., Frunbeis H. // Z. Naturforsch. 1982. V. 37B. N 11. P. 1472. doi 10.1515/znb-1982-1120

[18] Furuya K., Kawato K., Yokoyama H., Sakamoto A., Tasumi M. // J. Phys. Chem. A. 2003. V. 107. P. 8251-8258.

[19] Rademacher P., Marzinzik A.L., Kowski K., Weiß M.E. // Eur. J. Org. Chem. 2001. P. 121-130.

[20] Alaverdian I.S., Feofanov A.V., Gromov S.P., Vedernikov A.I., Lobova N.A., Alfimov M.V. // J. Phys. Chem. A. 2003. V. 107. P. 9542-9546.

[21] Saariahoa A.-M., Argyropoulos D.S., Jääskeläinen A.-S., Vuorinen T. // Vibrational Spectroscopy. 2005. V. 37. P. $111-121$.

[22] Kubicki A.A. // Chem. Phys. Lett. 2009. V. 483. N 4. P. 268-272.

[23] Choi Ch.H., Kertesz M. // J. Phys. Chem. A. 1997. V. 101. P. 3823-3831.

[24] Brédas J.L., Silbey R.J. // Conjugated polymers: the novel science and technology of highly conducting and nonlinear optically active materials. Dordrecht, Boston: Kluwer Academic Publishers, 1991. P. 414-425. 
[25] Molnar V., Billes F., Tyihak E., Mikosch H. // Spectrochim. Acta A. 2008. V. 69. P. 542-558.

[26] Nagano Ya, Ikoma T., Akiyama K., Tero-Kubota Sh. // J. Am. Chem. Soc. 2003. V. 125. P. 14103-14112.

[27] Osad'ko I.S. Selective Spectroscopy of Single Molecules. Berlin: Springer series in Chemical Physics. 2002. V. 69.; Осадько И.С. Селективная спектроскопия одиночных молекул. М.: Физматлит, 2000.

[28] Bouwman W.G., Jones A.C., Phillips D., Thibodeau P., Friel Ch., Christensen R.L. // J. Phys. Chem. 1990. V. 94. P. 7429-7434.

[29] Zhang A., Xiao Ch., Wu Ya., Li Ch., Ji Yu., Li L., Hu W., Wang Zh., Ma W., Li W. // Macromolecules. 2016. V. 49. P. 6431-6438.

[30] Katoh R., Hashimoto M., Takahashi A., Sonoda Y., Yago T., Wakasa M. // J. Phys. Chem. C. 2017. V. 121. N 46. P. 25666-25671.

[31] Sonoda Y., Goto M., Tsuzuki S., Tamaoki N. // J. Phys. Chem. A. 2007. V. 111. P. 13441-13451.

[32] Weigel A., Ernsting N.P. // J. Phys. Chem. B. 2010. V. 114. N 23. P. 7879-7893.

[33] Marri E., Pannacci D., Galiazzo G., Mazzucato U., Spalletti A. // J. Phys. Chem. A. 2003. V. 107. N 50. P. $11231-11238$.

[34] Gierschner J., Mack H.-G., Lüer L., Oelkrug D. // J. Chem. Phys. 2002. V. 116. N 19. P. 8596-8609.

[35] Buch T.E., Scott G.W. // J. Chem. Phys. 1981. V. 85. N 2. P. 144-146.

[36] Erckel R., Frunbeis H. // Z. Naturforsch. 1982. B. 37B. N 11. S. $1472-1480$.

[37] Васильева И.А., Компанееи, В.В. // Опт. и спектр. 2013. T. 114. № 1. С. 65-71.

[38] Компанееи, В.В., Васильева И.А. // Опт. и спектр. 2017. T. 122. № 4. C. 635-644.

[39] Компанееи, В.В., Васильева И.А. // Известия РАН. Сер. физ. 2016. Т. 80. № 7. С. 927-933.

[40] Компанееи, В.В., Васильева И.А. // Опт. и спектр. 2013. T. 115. № 4. C. 563-571.

[41] Васильева И.А., Войтова Н.А., Нурмухаметов Р.Н. // Опт. и спектр. 2012. Т. 112. № 3. С. 488-497.

[42] Наумова Н.Л., Васильева И.А., Осадько И.С., Наумов А.В. // Опт. и спектр. 2005. Т. 98. № 4. С. 586-594.

[43] Singh A.K., Mahalaxmi G.R. // Photochemistry and Photobiology. 2000. V. 71. P. 387-396.

[44] Tsumura K., Furuya K., Sakamoto A., Tasumi M. // J. Raman Spectroscopy. 2008. V. 39. N 11. P. 1584-1591.

[45] Watanabe H., Okamoto Yu., Furuya K. // J. Phys. Chem. A. 2002. V. 106. N 14. P. 3318-3324.

[46] Gagliardi L., Orlandi G., Molina V., Malmqvist P.-A., Roos B. // J. Phys. Chem. A. 2002. V. 106. N 14. P. $7355-7361$. 